\title{
Maspin protein expression correlates with tumor progression in non-muscle invasive bladder cancer
}

\author{
MARIO W. KRAMER ${ }^{1 *}$, SANDRA WAALKES ${ }^{1 *}$, JÖRG HENNENLOTTER ${ }^{2}$, JÜRGEN SERTH ${ }^{1}$, \\ ARNULF STENZL ${ }^{2}$, MARKUS A. KUCZYK ${ }^{1}$ and AXEL S. MERSEBURGER ${ }^{1}$ \\ ${ }^{1}$ Department of Urology, Hannover Medical School, OE-6240, Carl-Neuberg-Str. 1, D-30625 Hannover; \\ ${ }^{2}$ Department of Urology, Eberhard Karls University, Hoppe-Seyler-Str. 3, D-72076 Tübingen, Germany
}

Received March 1, 2010; Accepted May 14, 2010

DOI: 10.3892/ol_00000110

\begin{abstract}
Maspin is a $42-\mathrm{kDa}$ protein that belongs to the family of serine protease inhibitors. It is involved in various physiological processes. In cancer tissue, Maspin was found to influence angiogenesis, tumor growth, metastasis and the prognosis of tumor patients. This study was performed to analyze the involvement of Maspin in transitional cell carcinoma of the bladder as well as its prognostic impact in a large patient cohort. Specimens from 162 non-muscle invasive bladder cancer patients (pTa, 91; pT1, 71) treated by transurethral resection with a minimum 3-year follow-up (median 58.5 months) were included in the present investigation. Tissue microarrays were constructed, and the specimens were immunohistochemically stained for Maspin protein expression. Each tissue specimen was assessed on a staining scale ranging from 0 (no staining) to 300 (strong staining) and correlated with various clinicopathological parameters. Maspin protein expression predicted progression with a sensitivity of $95 \%$ and a specificity of $70 \%(\mathrm{p}<0.001)$. In predicting recurrence, Maspin staining showed $52 \%$ sensitivity and $67 \%$ specificity $(\mathrm{p}<0.05)$. Kaplan-Meier analyses were performed, and a low Maspin protein expression was correlated with a higher incidence of tumor progression ( $\mathrm{p}<0.0001)$. However, expression levels of Maspin protein did not distinguish between pTa and pT1 specimens. Multivariate analyses indicated Maspin expression as an independent factor for predicting progression $(\mathrm{p}<0.0001)$ and recurrence $(\mathrm{p}<0.05)$. The present results suggest that the Maspin protein expression is an independent prognostic indicator for predicting recurrence and progression to muscle invasive disease. This study further emphasizes a
\end{abstract}

Correspondence to: Dr Axel S. Merseburger, Department of Urology, Hannover Medical School, Carl-Neuberg-Str. 1, OE 6240, D-30625 Hannover, Germany

E-mail: merseburger.axel@mh-hannover.de

*Contributed equally

Key words: biomarker, maspin, recurrence, prognosis, progression, transitional cell carcinoma, transitional bladder cancer possible clinical role of this novel tumor suppressor gene in transitional cell carcinoma of the bladder.

\section{Introduction}

The incidence of bladder cancer has shown a steady increase over the past decade with an estimated 72,000 newly diagnosed cases in 2009 compared to approximately 54,000 in 1999 in the US $(1,2)$. At primary contact with medical health care, the majority of patients present with non-muscle invasive stages of this disease (pTa and pT1) and are commonly treated with bladder-sparing procedures such as transurethral resection of the bladder. Clinicopathologic parameters, such as tumor stage and grade, concomitant carcinoma in situ (CIS) and multifocality are known to be predictors of recurrence, progression and survival. Investigations over the past years have increased the understanding of the biological behavior of this disease and have led to novel prognostic and therapeutic recommendations and approaches (3). However, more than 50 to $70 \%$ of non-muscle invasive bladder cancer cases recur within 3 years after primary treatment with a 10 to $30 \%$ progression rate to muscle invasive tumor stages (pT2-pT4) $(4,5)$.

Maspin, a 42-kDa non-serine protease inhibitory protein of the serpin family, has versatile biological functions. It is an epithelial-specific gene and is the only serpin whose absence is lethal in early embryogenesis $(6,7)$. Its less specific but broad functions indicate that Maspin may be the oldest component of the serpin family. Maspin plays a role in the inhibition of angiogenesis, thereby regulating tumor growth, invasion and metastasis by blocking VEGF-/bFGF-induced vascular endothelial cell migration (8). Recombinant Maspin (rMaspin) is produced by fusion with glutathione S-transferase. Investigations involving rMaspin as well as secreted Maspin may show various anti-tumor effects besides angiogenesis inhibition, such as restraint of tumor adhesion and motility, thereby also modulating invasion and metastasis. Urokinasetype plasminogen activator, urokinase-type plasminogen activator receptor and collagen type I and III were previously identified as extracellular targets that strengthen focal adhesion contacts $(9,10)$. Among its biological functions, Maspin was found to induce apoptosis by regulating Bcl-2 family proteins and/or interacting with Hsp90 $(11,12)$. 
The diverse functions of Maspin and its involvement in tumor development have resulted in an extensive number of investigational studies. Only four studies involving the role of Maspin in bladder cancer have been published thus far, with controversial results (13-16). The present study immunohistochemically evaluated the expression of Maspin to determine the correlations between tumor expression in non-muscle invasive transitional cell carcinoma of the bladder (TCC) and patient recurrence- and progression-free survival.

\section{Materials and methods}

Patients and specimens. Archival specimens from 162 non-muscle invasive bladder cancer patients treated by transurethral resection between 1993 and 2003 were included in the present investigation. The same cohort was validated in a previous biomarker study investigating galectin-3 (17). The study was approved by the Ethics Committee of Tübingen University. Table I shows details of patient and tumor characteristics. Follow-up information was available for all 162 patients with a minimum follow-up time of 3 years. In case of tumor recurrence or progression, tumor specimens were included regardless of the time when either recurrence or progression occurred. Recurrence was defined as the reappearance of tumors of the same stage. Progression was defined as tumor development from non-muscle to muscle invasive disease. For classification, the tumor, node and metastasis (TNM) staging system was used. The tumors were initially graded by pathologists according to the 1973 World Helath Organization grading system (18).

Tissue microarray and immunohistochemical analysis. Tumor specimens were fixed in formalin, dehydrated and embedded in paraffin. The paraffin blocks were cut into 4- $\mu \mathrm{m}$ sections. Hematoxylin and eosin staining was performed for each tumor specimen to validate tumor stage and grade. Tissue microarrays (TMAs) were constructed and stained as previously described (17,19-21). A commercially available Maspin antibody (monoclonal mouse antibody; NCL-Maspin, Novocastra Laboratories Ltd.) in a dilution of 1:500 was used. For the negative control, the primary anti-human Maspin antibody was replaced by non-immune mouse serum.

The TMA slides were reviewed and classified by two independent investigators (M.W.K. and A.S.M) in a blinded manner. For statistical analysis, the immunohistochemical staining reaction was classified according to a semiquantitative reference scale ranging from ' 0 ' to ' $3+$ ', depending on the intensity of the Maspin protein expression. The relative amount of tumor cells stained positively for Maspin $(0-100 \%)$ in conjunction with the rating of the staining intensity, resulted in a staining score ranging from 0 to 300 as previously described (19). The same evaluation method was validated in previous studies $(17,21)$. The concordance rate of the investigators was $90 \%$.

Statistical analysis. The JMP program was used for statistical evaluations. A D'Augostino and Pearson omnibus normality test was performed to determine whether all data sets were parametric or non-parametric. One-way ANOVA and the Student's t-test were applied to correlate the Maspin
Table I. Frequency distribution of clinicopathological parameters and average Maspin expression levels.

\begin{tabular}{lccc}
\hline $\begin{array}{l}\text { Clinicopathological } \\
\text { parameters }\end{array}$ & $\begin{array}{c}\text { No. of } \\
\text { patients }^{\mathrm{a}}\end{array}$ & $\%$ & $\begin{array}{c}\text { Maspin } \\
\text { expression } \\
\text { levels }\end{array}$ \\
\hline Clinical data & & & \\
Male & 119 & 73 & 176 \\
Female & 43 & 27 & 157 \\
Age, years & 67.3 (mean) & 69 (median) & 171 \\
Carcinoma in situ + & 8 & 5 & 122 \\
Multifocality (+) & 60 & 37 & 164 \\
Lymph node (+) & - & - & - \\
Pathological distribution & & & \\
pTa & 91 & 56 & 179 \\
pT1 & 71 & 44 & 168 \\
G1 & 59 & 36 & 161 \\
G2 & 90 & 56 & 195 \\
G3 & 13 & 8 & 102
\end{tabular}

Follow-up data

\begin{tabular}{lccc} 
Time to recurrence $^{\mathrm{b}}$ & 14.6 (mean) & 10 (median) & - \\
Time to progression $^{\mathrm{b}}$ & 23.1 (mean) & 12 (median) & \multicolumn{1}{c}{} \\
pTa+pT1 recurrence (+) & 84 & 52 & 154 \\
pTa+pT1 recurrence (-) & 78 & 48 & 191 \\
pTa recurrence (+) & 47 & 52 & 156 \\
pTa recurrence (-) & 44 & 48 & 196 \\
pT1 recurrence (+) & 37 & 52 & 153 \\
pT1 recurrence (-) & 34 & 48 & 184 \\
pTa+pT1 progression (+) & 28 & 17 & 82 \\
pTa+pT1 progression (-) & 134 & 83 & 195 \\
pTa progression (+) & 9 & 10 & 80 \\
pTa progression (-) & 82 & 90 & 195 \\
pT1 progression (+) & 19 & 27 & 83 \\
pT1 progression (-) & 52 & 73 & 193
\end{tabular}

${ }^{\mathrm{a}} \mathrm{n}=162$ (non-muscle invasive). ${ }^{\mathrm{b}}$ Time was assessed in months. ${ }^{\mathrm{c}}$ None of the patients with non-muscle invasive bladder cancer were assessed for positive lymph nodes.

expression with various tumor characteristics. Time-to-event probabilities were estimated by the univariate Kaplan-Meier method. The Cox proportional hazard model was applied for the multivariate analysis. $\mathrm{P}<0.05$ was considered to indicate statistically significant differences.

\section{Results}

Clinicopathological data. Specimens of 162 patients [119 (73\%) male and 43 (27\%) female] with non-muscle invasive TCC as detected using cystoscopy were included in the present study. Tumor stages according to the TNM system were determined as follows: pTa, $\mathrm{n}=91(56 \%)$ and $\mathrm{pT} 1$, $\mathrm{n}=71(44 \%)$. Since exclusively non-muscle invasive tumors were investigated, no information on lymph node invasion and distant metastasis was recorded. Of the 162 specimens, $59(36 \%)$ were graded as $\mathrm{G} 1,90(56 \%)$ as $\mathrm{G} 2$ and $13(8 \%)$ 
Table II. Univariate and multivariate analyses. ${ }^{\mathrm{a}}$

\begin{tabular}{|c|c|c|c|c|}
\hline Parameters & P-value & Chi square & Odds ratio & Standard error \\
\hline \multicolumn{5}{|l|}{$\begin{array}{l}\text { Univariate analyses: Student's t-test } \\
\text { Fit } Y \text { by } X(Y=\text { Maspin })\end{array}$} \\
\hline Gender & 0.180 & 1.58 & 1.00 & 0.00 \\
\hline Age & 0.580 & - & - & - \\
\hline Carcinoma in situ & $<0.050$ & 2.28 & 7.68 & 0.00 \\
\hline Multifocality & 0.410 & 1.68 & 1.31 & 0.00 \\
\hline Ta vs. T1 & 0.420 & 0.43 & 1.52 & 0.00 \\
\hline G1 vs. G2 & $<0.050$ & 4.42 & 4.28 & 0.00 \\
\hline G1 vs. G3 & $>0.050$ & 1.67 & 1.30 & 0.00 \\
\hline G2 vs. G3 & $<0.010$ & 7.12 & 9.77 & 0.00 \\
\hline Recurrence vs. non-recurrence $(\mathrm{Ta}+\mathrm{T} 1)$ & $<0.050$ & 5.16 & 4.57 & 0.00 \\
\hline Recurrence vs. non-recurrence (Ta) & $<0.050$ & 3.73 & 6.36 & 0.00 \\
\hline Recurrence vs. non-recurrence (T1) & $>0.050$ & 1.46 & 1.77 & 0.00 \\
\hline Progression vs. non-progression $(\mathrm{Ta}+\mathrm{T} 1)$ & $<0.001$ & 12.16 & 15.50 & 0.00 \\
\hline Progression vs. non-progression (Ta) & $<0.001$ & 10.96 & 12.24 & 0.00 \\
\hline Progression vs. non-progression (T1) & $<0.001$ & 10.36 & 11.59 & 0.00 \\
\hline \multicolumn{5}{|l|}{$\begin{array}{l}\text { Multivariate analysis: Cox proportional hazard } \\
\text { Censor: recurrence of } \mathrm{Ta} / \mathrm{T} 1 \text { tumors }\end{array}$} \\
\hline Maspin expression & $<0.05$ & 6.69 & - & 0.00 \\
\hline T-stage & 0.70 & 0.15 & - & 0.16 \\
\hline Grade & 0.17 & 3.50 & - & 0.29 \\
\hline Multifocality & 0.51 & 0.41 & - & 0.14 \\
\hline Carcinoma in situ & 0.25 & 1.35 & - & 0.36 \\
\hline Gender & 0.75 & 0.10 & - & 0.16 \\
\hline Age & 0.18 & 1.76 & - & 0.01 \\
\hline \multicolumn{5}{|l|}{$\begin{array}{l}\text { Multivariate analysis: Cox proportional hazard } \\
\text { Censor: progression of Ta/T1 tumors }\end{array}$} \\
\hline Maspin expression & $<0.0001$ & 26.53 & - & 0.00 \\
\hline T-stage & 0.2500 & 1.32 & - & 0.32 \\
\hline Grade & 0.7900 & 0.47 & - & 0.60 \\
\hline Multifocality & 0.0900 & 2.97 & - & 0.25 \\
\hline Carcinoma in situ & 0.8100 & 0.05 & - & 0.63 \\
\hline Gender & 0.8500 & 0.04 & - & 0.26 \\
\hline Age & 0.2100 & 2.12 & - & 0.13 \\
\hline
\end{tabular}

${ }^{\text {ap}} \mathrm{P}$-values of the univariate and multivariate analyses are indicated using the Student's t-test and the Cox proportional hazard model.

as G3. Follow-up data were available for patients with a median follow-up of 58.5 months. Tumor recurrence was observed in $84(52 \%)$ patients, and tumor progression in $28(17 \%)$ patients. The average time interval between initial treatment and time of recurrence was 14 (3-72) and 23 (3-79) months for tumor progression (Table I).

Maspin expression. Maspin protein was detected in the nucleus and cytoplasm of the TCC specimens. A clear shift from high to faint staining within the nucleus was observed with more aggressive forms of non-muscle invasive TCC specimens in terms of risk of recurrence and progression. In 12 muscle-invasive tumor tissues used as comparative samples, no nuclear staining was observed (unpublished data).
Statistical analysis of Maspin protein expression in the TMAs is shown in Table II. Urothelium-defined (pTa) and minimally invasive TCC (pT1) were not distinguished by immunohistochemically detected Maspin protein expression $(\mathrm{p}=0.42)$. TCCs with low and high tumor grades (G1 and G3) showed a lower Maspin expression compared to grade 2 tumors. CIS was noted in 8 patients. While the presence of CIS correlated with Maspin staining scores $(\mathrm{p}<0.05)$, multifocal tumors at the time of diagnosis showed no significant correlation $(\mathrm{p}=0.41)$.

Maspin expression in correlation with follow-up information. We evaluated whether the Maspin protein expression in nonmuscle invasive bladder cancer specimens predicts tumor 


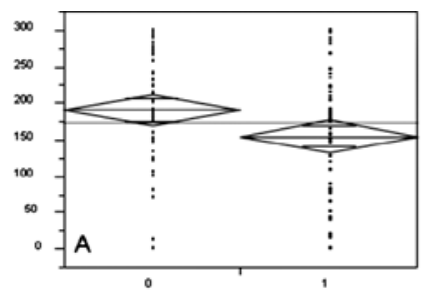

Recurrence [1-yes, 0-no]

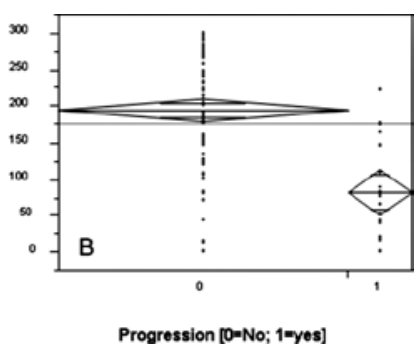

Figure 1. Maspin protein expression in $\mathrm{pTa} / \mathrm{pT} 1 \mathrm{TCC}$ cases in correlation with (A) tumor recurrence $(\mathrm{p}<0.05)$ and $(\mathrm{B})$ tumor progression $(\mathrm{p}<0.0001)$. The y-axis shows Maspin staining levels.

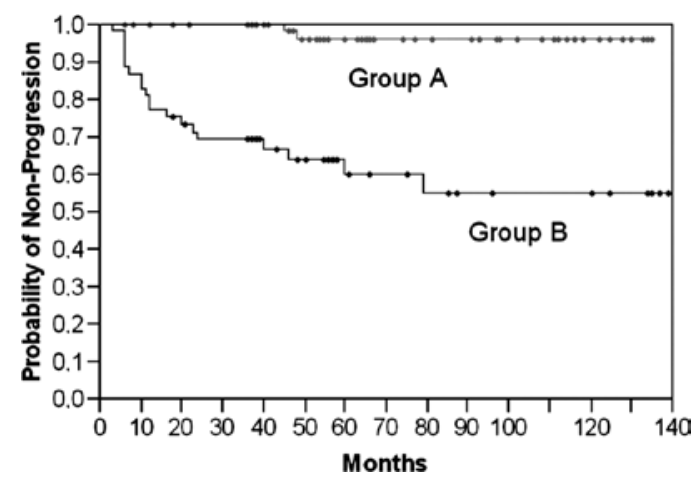

Figure 2. A decreased Maspin protein expression in pTa/pT1 TCC cases was significantly associated with an increased likelihood of tumor progression and a shorter progression-free survival (group B, Maspin level <175) in the Kaplan-Meier analysis $(\mathrm{p}<0.0001, \log -$ rank test). The $y$-axis shows the probability of non-progression.

recurrence and/or muscle invasion. Results are shown in Table II. Decreased staining levels in pTa, as well as in combination with $\mathrm{pT} 1$ tumors showed a higher incidence of tumor relapse $(\mathrm{p}<0.05$, Student's t-test) as confirmed by Chi-square analyses (Table II, Fig. 1). However, the Kaplan-Meier analysis did not indicate any significant prognostic value in respect to tumor recurrence. As shown in Table III, sensitivity and specificity were low, at 52 and $67 \%$, respectively. The univariate analysis of the prognostic significance of the staining score in relation to the likelihood of tumor progression showed that a strong Maspin expression and nuclear staining were associated with better survival in terms of tumor progression (Table II, Fig. 3). Significant lower staining scores were noted in pTa and pT1 tumors and a reduction in nuclear staining in tissue samples of patients who showed tumor progression within the follow-up time period ( $<<0.001$; Student's t-test). Subsequently, a Kaplan-Meier analysis was performed to further stratify the prognostic value of Maspin. Therefore, we classified the analyzed cohort into groups (A vs. B) according to a receiver operating curve. The cut-off limit (Group $A \geq 175$ vs. $\mathrm{B}<175)$ was based on the highest area under the curve and selected using the JMP statistical program (JMP 6 software, SAS Institute, Cary, NC, USA). Sensitivity and specificity were then calculated as 95 and $70 \%$, respectively (Table III). As illustrated in Fig. 2, low Maspin protein expression levels were highly associated with a shorter progression-free survival
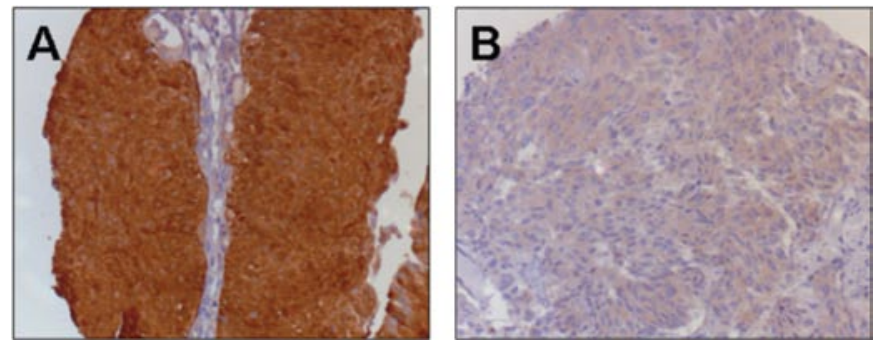

Figure 3. Maspin staining in TCC. (A) A strong Maspin protein expression with cytoplasmic and nuclear staining in non-recurrent and non-progressive pTa tumor. (B) Faint Maspin protein expression without nuclear staining in a progressive pTa tumor.

Table III. Sensitivities and specificities. ${ }^{\mathrm{a}}$

\begin{tabular}{lccc}
\hline & Sensitivity & Specificity & Log-rank value \\
\hline Recurrence & $52 \%$ & $67 \%$ & $\mathrm{p}=0.1820$ \\
Progression & $95 \%$ & $70 \%$ & $\mathrm{p}<0.0001$ \\
\hline
\end{tabular}

${ }^{a}$ Values for sensitivity and specificity of Maspin staining interference for predicting tumor recurrence and tumor progression are indicated. The cohort was divided into group A or B based on a Maspin staining score of 170. Refer also to the Kaplan-Meier analysis.

(46 vs. 18 months; $\mathrm{p}<0.0001$, Log-rank test). To determine which of the studied parameters and/or Maspin staining are independent prognostic indicators of tumor progression to muscle invasion, we used the Cox proportional hazard model. The statistical analysis affirmed only Maspin as an independent prognostic predictor for the likelihood of progression $(\mathrm{p}<0.0001$; Table II). Further parameters, such as tumor stage and grade, presence of concomitant CIS, multifocality, age and gender, were included. None of these parameters were statistically significant.

\section{Discussion}

Maspin is a $42-\mathrm{kDa}$ protein associated with various tumorrelated processes such as the inhibition of cell migration, cell invasion, angiogenesis, as well as improvement in cell adhesion and the induction of programmed cell death, thus classifying it as a tumor suppressor (8-12). Maspin expression has been observed in multiple tissues, e.g., epithelium of the breast, prostate, epidermis and lung (10,22-24). Localization within the cell appears to define its function. Loss of nuclear expression in ovarian, breast and lung cancer coincides with more aggressive phenotypes and decreased survival (25-27). We found a shift from nuclear to a predominantly cytoplasmic staining in 12 muscle-invasive tissue samples and, notably, in highly aggressive forms of non-muscle invasive bladder cancer. Tissues from patients that showed progression to muscle-invasive disease detected by subsequent cytoscopies following initial diagnosis showed less nuclear staining in the primary tissue. This observation stresses the assumption that cytoplasmic Maspin in the early steps of tumor progres- 
sion may signal an imperfect suppressive effect. Currently, only four studies evaluating Maspin expression in bladder cancer have been published. Of these, only Blandamura et al described the possible role of nuclear vs. cytoplasmic staining (16). These authors found a correlation between nuclear Maspin expression and lower histological grades (PUNLMP and low grade). This stresses the prognostic value of Maspin since low-grade and PUNLMP tumors are less likely to progress to muscle-invasive tumor stages $(\mathrm{pT} \geq 2)$.

By exclusively including pTa and pT1 tissues in this investigation, we were unable to show significance in respect to tumor stage and grade of the 162 examined samples. Similar results were published by Friedrich et al who found a predominantly weak Maspin protein expression in pTa and pT1 tumors without any correlation in respect to stage and grade (15). The two studies must be analyzed in contrast to that of Blandamura and colleagues who reported a statistical association between the Maspin staining pattern and stage and grade. It appears that a strong Maspin expression was associated with high-grade tumors whereas low-grade tumors and PUNLMP expressed less Maspin (16). When comparing Maspin protein expression in non-muscle invasive to muscle-invasive bladder cancer, Sugimoto et al were the first to describe an increased expression in higher tumor stages $(\mathrm{pT} \geq 2>\mathrm{pTa} / \mathrm{pT} 1)$ (14). However, in concordance to our results, no statistical difference was found when comparing pTa and pT1 tumors as 23 of the 27 specimens showed no Maspin expression at any rate.

Following loss of the Maspin protein expression in muscle-invasive tumor samples which suggested prognostic significance, Beecken and colleagues further examined Maspin mRNA expression in different bladder cell lines (13). Highly aggressive tumor cell lines (MGHU1, UMUC3) showed no Maspin mRNA expression. A subsequent investigation of the in vivo tumor growth rate found a close inverse correlation to the Maspin expression, highly emphasizing its prognostic value. However, apart from the previous promising results, no statistically significant correlation between Maspin staining and tumor relapse of pTa tumors was observed. Notably, the cohort of 24 patients for follow-up information in that study was small. In the present study, follow-up information was collected from 162 patients with a minimum follow-up time of 36 months (median 58.5). A Chi-square analysis indicated Maspin to be a prognostic indicator for tumor recurrence in $\mathrm{pTa}$ and $\mathrm{pTa} / \mathrm{pT} 1$ tumors $(\mathrm{p}<0.05)$, as well as an indicator for tumor progression in pTa, pT1 and pTa/pT1 TCC $(\mathrm{p}<0.001)$. However, the sensitivity and specificity of 52 and $67 \%$, respectively, for tumor recurrence were rather low compared to 95 and $70 \%$, respectively, for tumor progression. As described above, loss of Maspin appears to aggravate tumors via the deregulation of tumor cell growth, motility and angiogenesis. Accordingly, we found Maspin to be an independent prognostic indicator for tumor progression to muscle-invasive disease in TCC in a multivariate analysis $(\mathrm{p}<0.0001)$. Findings of a Kaplan-Meier analysis showed a shorter progression-free survival (18 vs. 46 months) for non-muscle invasive TCC with low Maspin protein expression scores.

In conclusion, the findings of the studies investigating the protein expression of Maspin in TCC indicated that no definitive answers can be formulated at this point. However, our study showed that Maspin may play a significant role in predicting tumor progression since Maspin was found to be an independent prognostic indicator. In order to promote diagnostic and therapeutic possibilities, additional studies are warranted to further clarify the role of Maspin in bladder cancer. Therefore, further investigational approaches must be engineered.

\section{References}

1. Landis SH, Murray T, Bolden S and Wingo PA: Cancer statistics. CA Cancer J Clin 49: 8-31 1999.

2. Jemal A, Siegel R, Ward E, Hao Y, Xu J and Thun MJ: Cancer statistics. CA Cancer J Clin 59: 225-249, 2009.

3. Guney S, Guney N, Canogullari Z and Ergenekon E: TaT1 low and intermediate transitional cell carcinoma of the bladder: recurrence rates and the timing of check cystoscopies within the first year. Urol Int 80: 124-128, 2008.

4. Holmang S, Hedelin H, Anderstrom C, Holmberg E, Busch C and Johansson SL: Recurrence and progression in low grade papillary urothelial tumors. J Urol 162: 702-707, 1999.

5. Herr HW: Tumor progression and survival of patients with high grade, noninvasive papillary (TaG3) bladder tumors: 15-year outcome. J Urol 163: 60-62, 2000.

6. Futscher BW, Oshiro MM, Wozniak RJ, et al: Role for DNA methylation in the control of cell type specific maspin expression. Nat Genet 31: 175-179, 2002.

7. Gao F, Shi HY, Daughty C, Cella N and Zhang M: Maspin plays an essential embryonic development. Development 131: 1479-1489, 2004.

8. Zhang M, Volpert O, Shi YH and Bouck N: Maspin is an angiogenesis inhibitor. Nat Med 6: 196-199, 2000.

9. Odero-Marah VA, Khalkhali-Ellis Z, Chunthapong J, et al: Maspin regulates different signaling pathways for motility and adhesion in aggressive breast cancer cells. Cancer Biol Ther 2: 398-403, 2003.

10. McGowen R, Biliran H Jr, Sager R and Sheng S: The surface of prostate carcinoma DU145 cells mediates the inhibition of urokinase-type plasminogen activator by maspin. Cancer Res 60: 4771-4778, 2000.

11. Jiang N, Meng Y, Zhang S, Mensah-Osman E and Sheng S: Maspin sensitizes breast carcinoma cells to induced apoptosis. Oncogene 21: 4089-4098, 2002.

12. Lockett J, Yin S, Li X, Meng Y and Sheng S: Tumor suppressive maspin and epithelial homeostasis. J Cell Biochem 97: 651-660, 2006.

13. Beecken WD, Engl T, Engels K, et al: Clinical relevance of maspin expression in bladder cancer. World J Urol 24: 338-344, 2006.

14. Sugimoto S, Maass N, Takimoto Y, et al: Expression and regulation of tumor suppressor gene maspin in human bladder cancer. Cancer Lett 203: 209-215, 2004.

15. Friedrich MG, Toma MI, Petri S, et al: Expression of maspin in non-muscle invasive bladder carcinoma: Correlation with tumor angiogenesis and prognosis. Eur Urol 45: 737-743, 2004.

16. Blandamura S, D'Alessandro E, Giacomelli L, et al: Expression of maspin in papillary Ta/T1 bladder neoplasms. Anticancer Res 28: 471-478, 2008

17. Kramer MW, Kuczyk MA, Hennenlotter J, et al: Decreased expression of galectin-3 predicts tumour recurrence in pTa bladder cancer. Oncol Rep 20: 1403-1408, 2008.

18. Mostofi FK, Sorbin L and Torloni H: Histological typing of urinary bladder tumours. International classification of tumours. World Health Organisation, Geneva, 1973.

19. Kramer MW, Merseburger AS, Hennenlotter J and Kuczyk M: Tissue microarrays in clinical urology - technical considerations. Scand J Urol Nephrol 41: 478-484, 2007.

20. Merseburger AS, Anastasiadis AG, Hennenlotter J, et al: Tissue microarrays: applications in urological cancer research. World J Urol 24: 579-584, 2006.

21. Merseburger AS, Kramer MW, Hennenlotter J, et al: Loss of galectin-3 expression correlates with clear cell renal carcinoma progression and reduced survival. World J Urol 26: 637-642, 2008.

22. Ngamkitidechakul C, Burke JM, O'Brien WJ and Twining SS: Maspin: synthesis by human cornea and regulation of in vitro stromal cell adhesion to extracellular matrix. Invest Ophthalmol Vis Sci 42: 3135-3141, 2001. 
23. Reis-Filho JS, Milanezi F, Silva P and Schmitt FC: Maspin expression in myoepithelial tumors of the breast. Pathol Res Pract 197: 817-821, 2001.

24. Reis-Filho JS, Torio B, Albergaria A and Schmitt FC: Maspin expression in normal skin and usual cutaneous carcinomas. Virchows Arch 441: 551-558, 2002.

25. Mohsin SK, Zhang M, Clark GM and Craig Allred D: Maspin expression in invasive breast cancer: Association with other prognostic factors. J Pathol 199: 432-435, 2003.
26. Sood AK, Fletcher MS, Gruman LM, et al: The paradoxical expression of maspin in ovarian carcinoma. Clin Cancer Res 8: 2924-2932, 2002.

27. Heighway J, Knapp T, Boyce L, et al: Expression profiling of primary non-small cell lung cancer for target identification. Oncogene 21: 7749-7763, 2002. 